Математички Билтен

ISSN 0351-336X (print)

43 (LXIX) No.1

ISSN 1857-9914 (online)

$2019(65-78)$

UDC: 515.122 .8

Скопје, Македонија

\title{
CONNECTEDNESS OF INVERSE LIMIT OF GENERALIZED TOPOLOGICAL SPACES
}

\author{
IVAN LONČAR
}

\begin{abstract}
The aim of this paper is to study the connectednes of inverse limit of generalized topological spaces introduced by Császár in [3.
\end{abstract}

\section{INTRODUCTION}

This section contains some basic definitions and propositions concerning supra topological and generalized topological spaces.

Definition 1. Let $J$ be any nonempty indexed set and let $\mathrm{X}$ be a nonempty set. A subfamily $\mu$ of $X$ is said to be supra topology on $X$ if:

i) $X, \emptyset \in \mu$

ii) If $A_{i} \in \mu$ for all $i \in J$, then $\cup A_{i} \in \mu$.

This definition can be reformulated as follows.

Definition 2. A subcollection $\mu \subset 2^{X}$ is called a supra topology on $X$, 6], if $X \in \mu$ and $\mu$ is closed under arbitrary union.

Definition 3. [10, Definition 2.3] Let $(X, \tau)$ be a topological space and $\mu$ be a supra topology on $X$. We call $\mu$ a supra topology associated with $\tau$ if $\tau \subseteq \mu$.

Proposition 1.1. [11, Theorem 1] If $\mu$ is a supra topology on $X$, then $T_{\mu}=\{A \subset X: A \cap B \in \mu$ for each $B \in \mu\}$ is a topology and $T_{\mu} \subset \mu$.

2010 Mathematics Subject Classification. Primary 54A05, Secondary: 54B35.

Key words and phrases. Generalized topological spaces, weakly compact. 
Definition 4. $(X, \mu)$ is called a supra topological space. The elements of $\mu$ are said to be supra open in $(X, \mu)$ and the complement of a supra open set is called a supra closed set.

Császár in [3] introduced the notion of generalized topological space (GTS) as another name for supra topology. He also introduced the notion of $\left(\mu_{1}, \mu_{2}\right)$-continuous function on GTS's and the separation axioms defined by replacing open sets by members of a GTS.

In general, let $M_{\mu}$ denote the union of all elements of $\mu$; of course, $M_{\mu} \in \mu$, and $M_{\mu}=X$ if and only if $\mu$ is a strong general topology (strong GT). A subset $A$ of $X$ is called $\mu$-open if $A \in \mu$. A subset $B$ of $X$ is called $\mu$-closed if $X \backslash B \in \mu$.

A point $x \in X$ is called a $\mu$-cluster point of $A$ if $U \cap(A \backslash\{x\}) \neq \emptyset$ for each $U$ in $\mu$ such that $x \in U$.

Let $\mathcal{B} \subset \exp X$ satisfy $\emptyset \in \mathcal{B}$. Then all unions of some elements of $\mathcal{B}$ constitute a GT $\mu(\mathcal{B})$, and $\mathcal{B}$ is said to be a base for $\mu(\mathcal{B})$.

Definition 5. [12, Definition 2.1] Let $X$ be a space. Then $\mu_{x}=\{U: x \in$ $U \in \mu\}$.

Definition 6. [12, Definition 2.2] Let $(X, \mu)$ be a GTS. $X$ is called a $\mu T_{2^{-}}$ space if $X$ satisfies the following $\mu T_{2}$-separation conditions: If $x, y \in X$ and $x \neq y$, then there are $U_{x} \in \mu_{x}$ and $U_{y} \in \mu_{y}$ such that $U_{x} \cap U_{y}=\emptyset$.

Definition 7. Let $(X, \mu)$ be a generalized topological space. Then $X$ is called a $\mu T_{1}$-space if for $x_{1}, x_{2} \in M_{\mu}$ with $x_{1} \neq x_{2}$, there exist $U, V \in \mu$ such that $x_{1} \in U, x_{2} \notin U$ and $x_{2} \in V, x_{1} \notin V$.

Definition 8. Let $\mu$ be a GT on $X$. We say that $M \subset X$ is $\mu$-open if and only if $M \in \mu ; N \subset X$ is $\mu$-closed if and only if $X-N \in \mu$.

Definition 9. ([7]) If $A \subset X$ then $i_{\mu} A$ denotes the union of all $\mu$-open sets contained in $A$ and $c_{\mu} A$ is the intersection of all $\mu$-closed sets containing $A$.

Both $i_{\mu}$ and $c_{\mu}$ are idempotent operations (where the operation $\gamma$ is said to be idempotent if and only if $\gamma \gamma A=\gamma A$ for $A \subset X)$.

Proposition 1.2. For $A \subset X$ and $x \in X$, we have $x \in c_{\mu} A$ if and only if $x \in M \in \mu$ implies $M \cap A \neq \emptyset$. 
Definition 10. Let $\mu$ be a GT on $X, \mu^{\prime}$ a GT on $X^{\prime}$ and $f: X \rightarrow X^{\prime}$. We say that the map $f$ is $\left(\mu, \mu^{\prime}\right)$-continuous if and only if $M^{\prime} \in \mu^{\prime}$ implies $f^{-1}\left(M^{\prime}\right) \in \mu$, and $\left(\mu, \mu^{\prime}\right)$-open if and only if $M \in \mu$ implies $f(M) \in \mu^{\prime}$. If $f$ is bijective and $\left(\mu, \mu^{\prime}\right)$-continuous, moreover $f^{-1}$ is $\left(\mu^{\prime}, \mu\right)$-continuous, then it is natural to say that $f$ is a $\left(\mu, \mu^{\prime}\right)$-homeomorphism.

Let $X$ be a non-empty set and let $\mathcal{B}$ be a collection of subsets of $X$ with $\emptyset \in \mathcal{B}$. Then the collection of all possible unions of elements of $\mathcal{B}$ forms a GT $\mu(\mathcal{B})$ on $X$ and $\mathcal{B}$ is called a base for $\mu(\mathcal{B})$.

Now, let $A \neq \emptyset$ be an index set, $X_{a} \neq \emptyset$ for $a \in A$, and $X=\prod\left\{X_{a}: a \in\right.$ $A\}$ the Cartesian product of the sets $X_{a}$. We denote by $p_{a}$ the projection $p_{a}: X \rightarrow X_{a}$.

Definition 11. ([4]) Suppose that, for $a \in A, \mu_{a}$ is a given GT on $X_{a}$. Let us consider all sets of the form $\prod\left\{M_{a}: M_{a} \in \mu_{a}\right\}$ and, with the exception of a finite number of indices $a, M_{a}=X_{a}$. We denote by $\mathfrak{B}$ the collection of all these sets. Clearly $\emptyset \in \mathfrak{B}$ so that we can define a GT $\mu=\mu(\mathfrak{B})$ having $\mathfrak{B}$ for base. We call $\mu$ the product of the GT s $\mu_{a}$ and denote it by $P_{a} \mu_{a}$.

The base for $\prod\left\{X_{a}: a \in A\right\}$ described in above Definition is called the canonical base for the Cartesian product. If each $\mu_{a}$ is a topology then clearly $\mu$ is the product topology of the factors $\mu_{a}$.

Proposition 1.3. If $B \in \mathfrak{B}$, then there exist a finite number of indices $a_{1}, a_{2}, \ldots, a_{n}$ such that $B=p_{a_{1}}^{-1}\left(M_{a_{1}}\right) \cap p_{a_{2}}^{-1}\left(M_{a_{2}}\right) \cap \ldots \cap p_{a_{2}}^{-1}\left(M_{a_{2}}\right)$.

Let us write $i=i_{\mu}, c=c_{\mu}, i_{k}=i_{\mu_{k}}, c_{k}=c_{\mu_{k}}$.

Consider in the following $A_{k} \subset X_{k}, A=\prod_{k \in K} A_{k}, x \in X$ and $x_{k}=$ $p_{k}(x)$.

Proposition 1.4. [4, Proposition 2.1.] $i A \subset \prod_{k \in K} i_{k} A_{k}$.

Proof. If $x \in i A$ then there is $M \in \mu$ such that $x \in M \subset A$. Then there are sets $M_{k} \in \mu_{k}$ such that $x \in \prod_{k \in K} M_{k} \subset M \subset A$. For $p_{k}(x)=x_{k}$, we have $x_{k} \in M_{k}$ so that $M_{k} \neq \emptyset$ and therefore $\prod_{k \in K} M_{k} \subset \prod_{k \in K} A_{k}$ implies $M_{k} \subset A_{k}$ for each $k$. Thus $x_{k} \in M_{k} \subset A_{k}$ shows that $x_{k} \in i_{k} A_{k}$.

Similarly, we have the following result.

Proposition 1.5. [3, Proposition 2.3.] $c A=\prod_{k \in K} c_{k} A_{k}$.

Proposition 1.6. 4, Proposition 2.4] The projection $p_{k}$ is $\left(\mu, \mu_{k}\right)$-open. 
In general, $p_{k}$ need not be $\left(\mu, \mu_{k}\right)$ continuous.

Proposition 1.7. [4, Proposition 2.7] If every $\mu_{k}$ is strong then $\mu$ is strong and $p_{k}$ is $\left(\mu, \mu_{k}\right)$ - continuous for $k \in K$.

\section{INVERSE SYSTEMS AND LIMITS}

Let $A$ be a set directed by the relation $\leq$ and let $\left\{X_{a}: a \in A\right\}$ be a family of sets indexed by $A$. For each pair $(a, b)$ of elements of $A$ such that $a \leq b$, let $p_{a b}$ be a mapping of $X_{b}$ into $X_{a}$, i.e. $p_{a b}: X_{b} \rightarrow X_{a}$. Suppose that $p_{a b}$ satisfy the following conditions [2, p. 191]:

$(\mathbf{L P} \mathrm{I})$ : The relations $a \leq b \leq c$ imply $p_{a c}=p_{a b} p_{b c}$,

$\left(\mathbf{L P}_{\mathrm{II}}\right)$ : For each $a \in A, p_{a a}$ is the identity mapping of $X_{a}$.

Then we say that $\mathbf{X}=\left\{X_{a}, p_{a b}, A\right\}$ is an inverse system of sets $X_{a}$ and bonding mapping $p_{a b}$.

Let $Y=\Pi\left\{X_{a}: a \in A\right\}$ be the product of the family of sets $\left\{X_{a}: a \in A\right\}$ and $X$ denote the subset of $Y$ consisting of all $x=\left(x_{a}: a \in A\right)$ (called a thread of $\mathbf{X})$ which satisfy each of the relation $x_{a}=p_{a b}\left(x_{b}\right)$ for each pair of indices $(a, b)$ such that $a \leq b$. The set $X$ is called inverse limit of the inverse system $\mathbf{X}=\left\{X_{a}, p_{a b}, A\right\}$ and is denoted by $\lim \mathbf{X}$ or by $\lim \left\{X_{a}, p_{a b}, A\right\}$.

Proposition 2.1. [5, 2.5.1. Proposition] The limit of an inverse system $\mathbf{X}=\left\{X_{a}, p_{a b}, A\right\}$ of Hausdorff spaces $X_{a}$ is the closed subset of the Cartesian product $\Pi\left\{X_{a}: a \in A\right\}$.

This Proposition is true for inverse systems $\mathbf{X}=\left\{X_{a}, p_{a b}, A\right\}$ of generalized spaces.

Proposition 2.2. The limit of an inverse system $\mathbf{X}=\left\{X_{a}, p_{a b}, A\right\}$ of $\mu T_{2}$ generalized topological spaces $X_{a}$ is the closed subset of the Cartesian product $\Pi\left\{X_{a}: a \in A\right\}$.

Proof. Let us prove that each point $x=\left\{x_{a}: a \in A\right\} \notin \lim \mathbf{X}$ has a neighbourhood which is disjoint with $\lim \mathbf{X}$. From $\left\{x_{a}: a \in A\right\} \notin \lim \mathbf{X}$ it follows that there exist a $b \in A$ such that for $c \leq b$ we have $p_{b c}\left(x_{b}\right) \neq x_{c}$. There exist disjoint $\mu$-open sets $U_{c}$ and $V_{c}$ such that $x_{c} \in U_{c}$ and $p_{b c}\left(x_{b}\right) \in$ $V_{c}$. Now $U_{b}=p_{b c}^{-1}\left(U_{c}\right) \cap p_{b c}^{-1}\left(V_{c}\right)$ is $\mu$-open set containing $x_{b}$. It follows that $U=p_{b}^{-1}\left(U_{b}\right)$ is an $\mu$-open set containing $x=\left\{x_{a}: a \in A\right\}$. Let us prove $U \cap \lim \mathbf{X}=\emptyset$. If $y=\left\{y_{a}: a \in A\right\} \in U$ then $p_{b}(y) \in U_{b}=p_{b c}^{-1}\left(U_{c}\right) \cap p_{b c}^{-1}\left(V_{c}\right)$. 
It follows that $p_{b c}\left(y_{b}\right) \neq y_{c}$. Thus, $y \notin \lim \mathbf{X}$. This means that $U \cap \lim \mathbf{X}=\emptyset$ and the proof is completed.

Let $\mathbf{X}=\left\{X_{a}, p_{a b}, A\right\}$ be an inverse system of generalized topological spaces and let $X=\lim \mathbf{X}$. For every $a \in A$ there is a continuous mapping $p_{a}=P_{a} \mid X: X \rightarrow X_{a}$, where $P_{a}: \Pi\left\{X_{a}: a \in A\right\} \rightarrow X_{a}$ is the projection,

Definition 12. Let $U \in \mu$ so that $x \in U$ and $U$ is contained in every $V \in \mu$ with $x \in V$. In this case we call $x$ a representative element for $U$.

Definition 13. A space $X$ that every $x \in X$ is a representative element for some $U \in \mu_{x}$ is called a $C_{0}$-space in [12].

Proposition 2.3. The family of all sets $p_{a}^{-1}\left(U_{a}\right)$, where $U_{a}$ is an $\mu$-open subset of $X_{a}$ and runs over a subset $A^{l}$ cofinal in $A$, is a base for the limit of the inverse system $\mathbf{X}=\left\{X_{a}, p_{a b}, A\right\}$. Moreover, if for every $a \in A$ base $B_{a}$ for $X_{a}$ is fixed, then the subfamily consisting of those $p_{a}^{-1}\left(U_{a}\right)$ in which $U_{a} \in B_{a}$, also is a base.

\section{QuASI-COMPACT GENERALIZED TOPOLOGICAL SPACES}

Definition 14. A generalized topological space $(X, \mu)$ is $\mu$-quasi-compact if every cover of $\mu$-open subsets of $(X, \mu)$ has the finite subcover.

Theorem 1. A generalized topological space $(X, \mu)$ is $\mu$-quasi-compact if and only if every family of $\mu$-closed subsets of $(X, \mu)$ which has the finite intersection property has non-empty intersection.

Let $\mathbf{X}=\left\{X_{a}, p_{a b}, A\right\}$ be an inverse system such that for every $a \in A$ there exists a family $\mathcal{S}_{a}$ of subsets of $X_{a}$ with the following properties, [2, p. 190]:

(I) Arbitrary intersection of the sets from $\mathcal{S}_{a}$ is a set from $\mathcal{S}_{a}$,

(II) If a family of subsets $\mathcal{F} \subset \mathcal{S}_{a}$ has the finite intersection property, then $\cap\{M: M \in \mathcal{F}\}$ is non-empty,

(III) $p_{a b}^{-1}\left(x_{a}\right) \in \mathcal{S}_{b}$ for every $x_{a} \in X_{a}$ and for every pair $a, b, a \leq b$,

$(\mathbf{I V}) p_{a b}\left(M_{b}\right) \in \mathcal{S}_{a}$ for every $M_{b} \in \mathcal{S}_{b}$ and for every pair $a, b, a \leq b$.

Theorem 2. Let $\mathbf{X}=\left\{X_{a}, p_{a b}, A\right\}$ be an inverse system which satisfies conditions $(\mathbf{I}),(\mathbf{I I}),(\mathbf{I I I})$ and $(\mathbf{I V})$. If $X=\lim \mathbf{X}$, then:

a) For all $a \in A$

$$
p_{a}(X)=\cap\left\{p_{a b}\left(X_{b}\right): b \geq a\right\}, a \in A,
$$


b) If $X_{a} \neq \emptyset$ for every $a \in A$, then $X \neq \emptyset$.

Proof. Let $S_{a}$ be a family of all non-empty subsets of $X_{a}$ and let $\mathcal{Y}$ be a family of all collections $Y=\left\{Y_{a}: Y_{a} \in S_{a}, a \in A\right\}$ such that $p_{a b}\left(Y_{b}\right) \subset Y_{a}$. The family $\mathcal{Y}$ is non-empty since $\mathbf{X} \in \mathcal{Y}$. For two collections $Y=\left\{Y_{a}\right.$ : $\left.Y_{a} \in S_{a}, a \in A\right\}$ and $Z=\left\{Z_{a}: Z_{a} \in S_{a}, a \in A\right\}$ we shall write $Y \geq Z$ if $Y_{a} \subset Z_{a}$ for every $a \in A$. It is clear that $(\mathcal{Y}, \geq)$ is a partially ordered set. The remaining part of the proof consists of several steps.

Step 1. There exists a maximal element in $(\mathcal{Y}, \geq)$.

It suffices to prove that $(\mathcal{Y}, \geq)$ is inductive, i.e. if $L=\left\{Y^{\lambda}: \lambda \in \Lambda\right\}$ is a strictly increasing chain in $(\mathcal{Y}, \geq)$, then there is an element $M \in(\mathcal{Y}, \geq)$ such that $M \geq Y^{\lambda}$ for every $\lambda \in \Lambda$. We define $M=\left\{M_{a}: M_{a} \in S_{a}, a \in A\right\}$ such that $M_{a}=\cap\left\{Y_{a}^{\lambda}: \lambda \in \Lambda\right\}$. From the properties (I) and (II) it follows that the set $M_{a}$ is non-empty $\mathcal{S}_{a}$ subset of $X_{a}$. Moreover, $p_{a b}\left(M_{b}\right) \subset M_{a}$.

Step 2. If $Y=\left\{Y_{a}: Y_{a} \in \mathcal{S}_{a}, a \in A\right\}$ is a maximal element of $(\mathcal{Y}, \geq)$, then $Y_{a}=p_{a b}\left(Y_{b}\right)$ for every pair $a, b \in A$ such that $a \leq b$.

Let $Z=\left\{Z_{a}: Z_{a} \in \mathcal{S}_{a}, a \in A\right\}$ be a collection such that $Z_{a}=\cap\left\{p_{a b}\left(Y_{b}\right)\right.$ : $b \geq a\}$. Each $p_{a b}\left(Y_{b}\right) \in \mathcal{S}_{a}$ since $Y_{b} \in \mathcal{S}_{b}$. From the properties (I) and (II) it follows that the set $Z_{a}$ is non-empty $S_{a}$ subset of $X_{a}$. In order to prove that $Z \in(\mathcal{Y}, \geq)$ it suffices to prove that $p_{a b}\left(M_{b}\right) \subset M_{a}$. If $a \leq b$ then $p_{a b}\left(Z_{b}\right) \subset \cap\left\{p_{a b}\left(p_{b c}\left(Y_{c}\right)\right): b \leq c\right\}=\cap\left\{p_{a c}\left(Y_{c}\right): c \geq b\right\}$. On the other hand, for every $d \geq a$ there is a $c \in A$ such that $c \geq b$, $d$. It follows that $p_{a c}\left(Y_{c}\right) \subset p_{a d}\left(Y_{d}\right)$. This means that

$$
\cap\left\{p_{a c}\left(Y_{c}\right): c \geq b\right\}=\cap\left\{p_{a d}\left(Y_{d}\right): c \geq b\right\}=Z_{a} .
$$

Finally, we have $Z \in(\mathcal{Y}, \geq)$. Moreover, $Z_{a} \subset Y_{a}$ for each $a \in A$. This means that $Z=Y$ since $Y$ is maximal.

Step 3. If $Y=\left\{Y_{a}: Y_{a} \in \mathcal{S}_{a}, a \in A\right\}$ is a maximal element of $(\mathcal{Y}, \geq)$, then $Y_{a}$ is one-point set for every $a \in A$.

Let $x_{a} \in Y_{a}$. Define

$$
Z_{b}=\left\{\begin{array}{cl}
Y_{b} \cap p_{a b}^{-1}\left(x_{a}\right), & \text { if } b \geq a, \\
Y_{b}, & \text { if } b \not \leq a .
\end{array}\right.
$$

Let us prove that $Z=\left\{Z_{a}: Z_{a} \in \mathcal{S}_{a}, a \in A\right\}$. We infer that each $Y_{b} \cap$ $p_{a b}^{-1}\left(x_{a}\right)$ is in $\mathcal{S}_{a}$. It is easy to prove that $p_{a b}\left(Z_{b}\right) \subset Z_{a}$. Hence, $Z \in(\mathcal{Y}, \geq)$. Now, $Z=Y$ since $Z \geq Y$ and $Y$ is maximal. This means $Y_{a}=\left\{x_{a}\right\}$. 
Step 4. $\lim \mathbf{X}$ is non-empty.

From Step 3 we have that $Z=\left\{Z_{a}: Z_{a} \in \mathcal{S}_{a}, a \in A\right\}=\left\{x_{a}: a \in A\right\}$ such that $p_{a b}\left(x_{b}\right)=x_{a}$ for every pair $a, b$ such that $b \geq a$.

Step 5. Let us prove that $p_{a}(X)=\cap\left\{p_{a b}\left(X_{b}\right): b \geq a\right\}$.

It is clear that $p_{a}(X) \subset \cap\left\{p_{a b}\left(X_{b}\right): b \geq a\right\}$. Let us prove that $p_{a}(X) \supset$ $\cap\left\{p_{a b}\left(X_{b}\right): b \geq a\right\}$. Let $x_{a} \in \cap\left\{p_{a b}\left(X_{b}\right): b \geq a\right\}$. This means that $Y_{b}=p_{a b}^{-1}\left(x_{a}\right)$ is non-empty for each $b \geq a$. Moreover, $Y_{b} \in \mathcal{S}_{b}$. For each $b$ non-comparable with $a$, let $Y_{b}=X_{b}$. Now, we have a collection $Y=\left\{Y_{a}\right.$ : $\left.Y_{a} \in \mathcal{S}_{a}, a \in A\right\}$ which is evidently in $(\mathcal{Y}, \geq)$. There exists a maximal element $Z=\left\{Z_{a}: Z_{a} \in \mathcal{S}_{a}, a \in A\right\}$ in $(\mathcal{Y}, \geq)$ such that $Z \geq Y$. It follows that each $Y_{a}$ is some $Z_{a}$ which is a point $z_{a} \in X_{a}$ (Step 3) since $Z$ is maximal. The collections $\left(z_{a}\right)$ is a point of $\lim \mathbf{X}$. Hence, $p_{a}(X)=\cap\left\{p_{a b}\left(X_{b}\right): b \geq a\right\}$.

Theorem 3. Let $\mathbf{X}=\left\{X_{a}, p_{a b}, A\right\}$ be an inverse system of non-empty $\mu$-quasi-compact $\mu T_{1}$ spaces $X_{a}$ and $\mu$-closed mappings $p_{a b}$. Then $\lim \mathbf{X}$ is non-empty.

Proof. Let $\mathcal{S}_{a}$ be a family of $\mu$-closed subsets of $X_{a}$ and let each $p_{a b}$ be a closed mapping. Now, we have the following properties:

(I) arbitrary intersection of $\mu$-closed sets from $\mathcal{S}_{a}$ is a $\mu$-closed set from $\mathcal{S}_{a}$, since arbitrary union of the $\mu$-open sets is a $\mu$-open.

(II) If a family of subsets $\mathcal{F} \subset \mathcal{S}_{a}$ has the finite intersection property, then $\cap\{M: M \in \mathcal{F}\}$ is non-empty since each $X_{a}$ is $\mu$-quasi-compact.

(III) $p_{a b}^{-1}\left(x_{a}\right) \in \mathcal{S}_{b}$ for every $x_{a} \in X_{a}$ and for every pair $a, b, a \leq b$, since in $T_{1}$ space each point is closed.Thus, is $p_{a b}^{-1}\left(x_{a}\right) \mu$-closed.

(IV) $p_{a b}\left(M_{b}\right) \in \mathcal{S}_{a}$ for every $M_{b} \in \mathcal{S}_{b}$ and for every pair $a, b, a \leq b$ since $p_{a b}$ is $\mu$-closed.

Using Theorem 2 we complete the proof.

Remark 3.1: In fact, from the proof of Theorem 2 it follows that each closed subsystem $\mathbf{Z}$ contains some minimal closed subsystem $\mathbf{Y}$.

Now we prove the quasi-compactness of the limit space.

Theorem 4. Let $\mathbf{X}=\left\{X_{\alpha}, p_{\alpha \theta}, A\right\}$ be an inverse system of quasi- compact $\mu T_{1}$ spaces $X_{\alpha}$ and $\mu$-closed mappings $p_{\alpha \beta}$. Then $\lim \mathbf{X}$ is $\mu$-quasicompact. 
Proof. Let $\mathcal{U}=\left\{U_{\mu}: \mu \in M\right\}$ be an $\mu$-open cover of $\lim \mathbf{X}$. By virtue of the definition of a base in $\lim \mathbf{X}$ there is an open set $U_{\mu, a} \subseteq X_{\alpha}$, for each $\alpha \in A$ and $\mu \in M$, such that $U_{\mu}=\Pi\left\{U_{\mu, \alpha}: \alpha \in A\right\}, p_{\alpha}^{-1}\left(U_{\mu, \alpha}\right) \subseteq U_{\mu}$ and $U_{\mu, \alpha}$ is a maximal set with respect to property $p^{-1}\left(U_{\mu, \alpha}\right) \subseteq U_{\mu}$. Let $\mathcal{U}_{\alpha}$ be a family $\left\{U_{\mu, \alpha} \backslash \cdot \alpha \in A\right\}$. If $\mathcal{U}_{\alpha}$ is the cover of $X_{a}$ then $p_{\alpha}^{-1}\left(\mathcal{U}_{\alpha}\right)$ is a cover $\lim \mathbf{X}$ which refines $\mathcal{U}$. This means that $\mathcal{U}$ has a finite subcover since $\mathcal{U}_{\alpha}$ has a finite subcover. Now we prove that there exists an $\alpha \in A$ such $\mathcal{U}_{\alpha}$ is a cover of $X_{\alpha}$. In the opposite case the set $Z_{\alpha}=X_{\alpha} \backslash\left(\cup\left\{U_{\mu \alpha}: \mu \in M\right\}\right\rangle$ is non-empty for each $\alpha \in A$. Now we obtain a closed subsystem $\mathbf{Z}=\left\{Z_{\alpha}, p_{a \beta} \mid Z_{\beta}, A\right\}$. By virtue of Remark 3.1 it follows that there is a closed subsystem $\mathbf{Y} \leq \mathbf{Z}$ such that $\mathbf{Y}$ is minimal. From the proof of Theorem 2 it follows that $\lim \mathbf{Y}$ is non-empty. This means that $\lim \mathbf{Z} \neq \emptyset$. Let $z$ be any point of $\lim \mathbf{Z}$. It is easy to prove that $z \notin \cup\left\{f_{\alpha} \rightarrow 1\left(U_{\mu, \alpha}\right): \alpha \in A, \mu \in M\right\}$. This is impossible since $\mathcal{U}=\left\{U_{\mu}: \mu \in M\right\}$ is the cover of $\lim \mathbf{X}$. Thus, there exists an $\alpha \in A$ such $\mathcal{U}_{\alpha}$ is a cover of $X_{\alpha}$. The proof is complete.

\section{Connectedness of inverse limit of GENERALIZED CONNECTED SPACES}

Definition 15. [1, Definition 3.1] Let $(X, \mu)$ be a GTS. $X$ is called $\mu$ connected if there are no nonempty disjoint $\mu$-open subsets $U, V$ of $X$ such that $U \cup V=X$.

Theorem 5. A generalized topological space $(X, \mu)$ is $\mu$-quasi-compact if and only if every family of $\mu$-closed subsets of $(X, \mu)$ which has the finite intersection property has non-empty intersection.

Let us recall, [1, that if $(X, \mu)$ GTS then $X$ is called $\mu$-connected if there are no nonempty disjoint $\mu$-open subsets $U, V$ of $X$ such that $U \cup V=X$.

Corollary 5.1. It is clear that $X$ is $\mu$-connected if and only if there are no nonempty disjoint $\mu$-closed subsets $F_{1}, F_{2}$ of $X$ such that $F_{1} \cup F_{2}=X$.

We shall prove the following result.

Theorem 6. Let $\mathbf{X}=\left\{X_{a}, p_{a b}, A\right\}$ be an inverse system of $\mu$-quasicompact spaces $X_{a}$ such that $X=\lim \mathbf{X}$ is $\mu$-quasi-compact and surjective projections $p_{a}: X \rightarrow X_{a}$. Then $\lim \mathbf{X}$ is $\mu$-connected if each $X_{a}$ is $\mu$-connected. 
Proof. Suppose that $X$ is not $\mu$-connected. It follows that there exist $\mu$ closed sets $F_{1}, F_{2}$ such that

$$
F_{1} \cup F_{2}=X
$$

and

$$
F_{1} \cap F_{2}=\emptyset
$$

Consider the sets

$$
Y_{a}=c_{\mu}\left(p_{a}\left(F_{1}\right)\right) \cap c_{\mu}\left(p_{a}\left(F_{2}\right)\right)
$$

From the ontoness of $p_{a}: X \rightarrow X_{a}$ it follows that

$$
c_{\mu}\left(p_{a}\left(F_{1}\right)\right) \cup c_{\mu}\left(p_{a}\left(F_{2}\right)\right)=X_{a} .
$$

This relations implies that $Y_{a} \neq \emptyset$ since from $Y_{a}=\emptyset$ it follows that $X_{a}$ is not $\mu$-connected.

Now the family $\left\{p_{a}^{-1}\left(Y_{a}\right): a \in A\right\}$ is the family with finite intersection property of $\mu$-closed subset of $\mu$-quasi-compact space $X=\lim \mathbf{X}$. Thus

$$
Y=\cap\left\{p_{a}^{-1}\left(Y_{a}\right): a \in A\right\} \neq \emptyset
$$

Let $y \in Y$. From the relations (1) and (2) that $y \in F_{1} / F_{2}$ or $y \in F_{2} / F_{1}$. In any case there exists $b \in A$ and a $\mu$-open set $V_{b} \subset X_{b}$ such that $p_{b}^{-1}\left(V_{b}\right) \cap$ $F_{2}=\emptyset$ or $p_{b}^{-1}\left(V_{b}\right) \cap F_{1}=\emptyset$. It follows that $p_{b}(y) \notin p_{b}\left(F_{2}\right)$ or $p_{b}(y) \notin p_{b}\left(F_{1}\right)$. We infer that $p_{b}(y) \notin Y_{b}$. This is impossible because (5). This means that $F_{1}=\emptyset$ or $F_{2}=\emptyset$, i.e. that $X$ is $\mu$-connected.

\section{Connectedness if inverse limit of Weakly $\mu$-COMpaCt SPACES}

Definition 16. A function $f:(X, \mu) \rightarrow(Y, \kappa)$ is called $(\mu, \kappa)$-continuous if the inverse image of each $\kappa$-open set is $\mu$-open.

Definition 17. Let $A$ be a nonempty subset of a space $(X ; \mu)$. The generalized subspace topology on $A$ is the collection $\{U \cap A: U \in \mu\}$, and will be denoted by $\mu_{A}$. The generalized subspace $A$ is the generalized topological space $\left(A, \mu_{A}\right)$.

Definition 18. [8, Definition 2.1.] A space $(X ; \mu)$ is called weakly $\mu$ compact (briefly $w \mu$-compact) if any $\mu$-open cover of $X$ has a finite subfamily, the union of the $\mu$-closures of whose members covers $X$.

It is clear that every $\mu$-compact space $(X ; \mu)$ is $w \mu$-compact. 
Lemma 1. [8, Proposition 2.7.] A space $(X, \mu)$ is wu-compact if and only if any $\mu$-regular open cover of $X$ has a finite subfamily, the union of the $\mu-$ closures of whose members covers $X$.

Lemma 2. [8, Proposition 2.8.] For a space $(X ; \mu)$, the following are equivalent:

(i) $X$ is wh-compact,

(ii) For any family $\mathcal{U}=\left\{U_{\alpha}: \alpha \in \Lambda\right\}$ of $\mu$-closed subsets of $X$ such that $\cap\left\{U_{\alpha}: \alpha \in \Lambda\right\}=\emptyset$, there exists a finite subset $\Lambda_{0}$ of $\Lambda$ such that $\cap\left\{i_{\mu}\left(U_{\alpha}\right): \alpha \in \Lambda_{0}\right\}=\emptyset$,

(iii) For any family $\mathcal{U}=\left\{U_{\alpha}: \alpha \in \Lambda\right\}$ of $\mu$-regular closed subsets of $X$ such that $\cap\left\{U_{\alpha}: \alpha \in \Lambda\right\}=\emptyset$, there exists a finite subset $\Lambda_{0}$ of $\Lambda$ such that $\cap\left\{i_{\mu}\left(U_{\alpha}\right): \alpha \in \Lambda_{0}\right\}=\emptyset$.

Definition 19. [8, Definition 2.9.] Let $A$ be a subset of a space $(X, \mu)$. A point $x \in X$ is said to be a $\theta_{\mu}$-accumulation point of $A$ if $c_{\mu}(U) \cap A \neq \emptyset$ for every $\mu$-open subset $U$ of $X$ that contains $x$. The set of all $\theta_{\mu}$-accumulation points of $A$ is called the $\theta_{\mu}$-closure of $A$ and is denoted by $\left(c_{\mu}\right)_{\theta}(A) . A$ is said to be $\mu_{\theta}$-closed if $\left(c_{\mu}\right)_{\theta}(A)=A$. The complement of a $\mu_{\theta}$-closed set is called $\mu_{\theta^{-}}$open.

It is clear that $A$ is $\mu_{\theta}$-open if and only if for each $x \in A$, there exists a $\mu$-open set $U$ such that $x \in U \subset c_{\mu}(U) \subset A$.

Theorem 7. If $X$ is a $\mu$-space then:

(a) the empty set and the whole space are $\mu_{\theta}$-closed,

(b) arbitrary intersection of $\mu_{\theta}$-closed sets are $\mu_{\theta}$-closed,

(c) $c_{\mu}(K) \subset\left(c_{\mu}\right)_{\theta}(K)$ for each subset $K$,

(d) $a \mu_{\theta}$-closed subset is closed.

Lemma 3. If $X$ is a $\mu$-space, then for each $Y \subset X$ there exists a minimal $\mu_{\theta}$-closed subset $Z \subset X$ such that $Y \subset Z$.

Proof. The collection $\Phi$ of all $\mu_{\theta}$-closed subsets $W$ of $X$ which contains $Y$ is non-empty since $X \in \Phi$. By (b) of Theorem 7 we infer that $Z=\cap\{W$ : $W \in \Phi\}$ is a minimal $\mu_{\theta}$-closed subset $Z \subset X$ containing $Y$.

From Theorem 7 it follows that the family of all $\mu_{\theta}$-open subsets of $(X, \mu)$ is a new generalized topology (GT) $\mu_{\theta}$ on $X$. 
Definition 20. Let $(X, \mu)$ be a GT space. The $\mu_{\theta}$-space of $X$ is the space $\left(X, \mu_{\theta}\right)$.

In the sequel we shall use denotations $X_{\mu}$ and $X_{\mu_{\theta}}$.

Definition 21. Let $X$ be a space. Then $\mu_{x}=\{U: x \in U \in \mu\}$.

Lemma 4. If $X_{\mu}$ is a $\mu T_{2}$ space, then $X_{\mu_{\theta}}$ is $\mu T_{1}$-space.

Proof. Let $x$ be any point of $X_{\mu}$. For every another point $y \in X_{\mu}, y \neq x$, there exists a pair of $\mu$-open disjoint set $U, V$ (see 6) such such that $x \in U$ and $y \in V$. It follows that $U \cap c_{\mu}(V)=\emptyset$. We conclude that $x$ is $\mu_{\theta}$-closed and, consequently, $X_{\mu_{\theta}}$ is $\mu T_{1}$-space.

Proposition 5.1. If $X_{\mu}$ is a $\mu T_{2}$ and w $\mu$-compact space, then each family of $\mu_{\theta}$-closed subsets with the finite intersection property has non-empty intersection.

Proof. If $\left\{F_{\alpha}: \alpha \in \Lambda\right\}$ is a family of $\mu_{\theta}$-closed subsets with the finite intersection property, then $\left\{F_{\alpha}: \alpha \in \Lambda\right\}$ is a family of $\mu$-closed subsets of $X_{\mu_{\theta}}$ with the finite intersection property. Thus, $\cap\left\{F_{\alpha}: \alpha \in \Lambda\right\}$ is nonempty since $X_{\mu_{\theta}}$ is $\mu$-quasi-compact, [9].

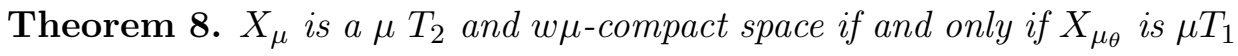
and $\mu$-compact space.

Proof. If. Let $X_{\mu_{\theta}}$ be $\mu T_{1}$ and $\mu$-compact space and let $\left\{F_{a}: a \in A\right\}$ be a family of $\mu_{\theta}$-closed subsets of $X_{\mu}$ with the finite intersection property. Now $\left\{F_{a}: a \in A\right\}$ is the family of $\mu$-closed subsets of $X_{\mu_{\theta}}$ which is $\mu T_{1}$ and $\mu$-compact space. It follows that the family $\left\{F_{a}: a \in A\right\}$ has non-empty intersection. Thus, $X_{\mu}$ is a $w \mu$-compact space.

Only if. If $\left\{F_{a}: a \in A\right\}$ is a family of $\mu$-closed subsets of $\left\{F_{a}: a \in A\right\}$ with the finite intersection property, then $\left\{F_{a}: a \in A\right\}$ is a family of $\mu_{\theta}$-closed subsets of $X_{\mu}$ with the finite intersection property. Since $X_{\mu}$ is $w \mu$-compact space, we infer that $\cap\left\{F_{a}: a \in A\right\}$ is non-empty. By this we infer that $X_{\mu_{\theta}} \mu$-compact space.

The following theorems are the main results of this section.

Theorem 9. Let $\mathbf{X}=\left\{X_{a}, p_{a b}, A\right\}$ be an inverse system of non-empty $w \mu$ compact $\mu T_{2}$ spaces and $\mu$-closed mappings $p_{a b}$. If $X=\lim X$, then: 
a) For all $a \in A$

$$
p_{a}(X)=\cap\left\{p_{a b}\left(X_{b}\right): b \geq a\right\},
$$

b) If $X_{a} \neq \emptyset$ for every $a \in A$, then $X \neq \emptyset$.

Proof. Now inverse system $\mathbf{X}=\left\{X_{a}, p_{a b}, A\right\}$ is inverse system

$$
\mathbf{X}_{\mu}=\left\{X_{a_{\mu_{\theta}}}, p_{a b}, A\right\}
$$

of $\mu$ - quasi-compact spaces and closed mappings $p_{a b}$. Using Theorem 2 we complete the proof.

Lemma 5. Let $\mathbf{X}=\left\{X_{a}, p_{a b}, A\right\}$ be an inverse system of $w \mu$-compact $\mu T_{2}$ non-empty spaces and $\mu$-closed surjective bonding mapping $p_{a b}$. Then the projections $p_{a}: \lim \mathbf{X} \rightarrow \mathbf{X}_{a}, a \in A$, are surjective and $\mu$-closed.

Proof. The inverse system $\mathbf{X}_{\mu}=\left\{X_{a_{\mu_{\theta}}}, p_{a b}, A\right\}$ satisfies the condition of Theorem 2 by which we complete the proof.

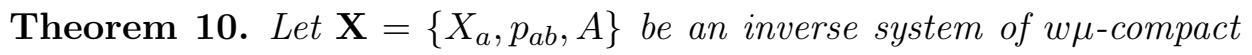
$\mu T_{2}$ non-empty spaces and $\mu$-closed surjective bonding mapping $p_{a b}$. Then $\lim \mathbf{X}$ is wu-compact.

Proof. By Theorem 2 the inverse system $\mathbf{X}_{\mu}=\left\{X_{a_{\mu_{\theta}}}, p_{a b}, A\right\}$ of $\mu$-quasicompact spaces has $\mu$-quasi-compact $\operatorname{limit} \lim \mathbf{X}_{\mu}$. This means that $\lim \mathbf{X}$ w $\mu$-compact space.

Definition 22. We say that the mapping $f: X \rightarrow Y$ has the inverse property if $f^{-1}\left(\mathrm{c}_{\mu} V\right)=\mathrm{c}_{\mu} f^{-1}(V)$ for every $\mu$-open set $V \subset Y$.

Now we shall prove the following result.

Theorem 11. Let $\mathbf{X}=\left\{X_{a}, p_{a b}, A\right\}$ be an inverse system of $w \mu$-compact spaces such that $\lim \mathbf{X}$ is w $\mu$-compact and the projections $p_{a}: \lim \mathbf{X} \rightarrow \mathbf{X}_{a}$, $a \in A$, are surjective with the inverse property. If the spaces $X_{a}$ are $\mu$ connected, then $X=\lim \mathbf{X}$ is $\mu$-connected.

Proof. Suppose that $X$ is not $\mu$-connected. It follows that there exist $\mu$ closed sets $F_{1}, F_{2}$ such that

$$
F_{1} \cup F_{2}=X
$$

and

$$
F_{1} \cap F_{2}=\emptyset .
$$


For each $a \in A$ let $U_{a} \subset X_{a}$ be a maximal $\mu$-open set such that $p_{a}^{-1}\left(U_{a}\right) \subset$ $F_{1}$. Similarly, let $V_{a} \subset X_{a}$ be a maximal $\mu$-open set such that $p_{a}^{-1}\left(V_{a}\right) \subset F_{2}$. From the $w \mu$-compactness of $X$ it follows that there exist finitely many $U_{a 1}, \ldots, U_{a n}, V_{b 1}, \ldots, V_{b m}$

$$
X=\cup_{i=1}^{n} c_{\mu}\left(p_{a i}^{-1}\left(U_{a i}\right)\right) \cup\left(\cup_{j=1}^{m} c_{\mu}\left(p_{b j}^{-1}\left(U_{b j}\right)\right)\right) .
$$

We may use that $a_{i}=b_{j}$. This means that

$$
X=\cup_{i=1}^{n} c_{\mu}\left(p_{a i}^{-1}\left(U_{a i}\right)\right) \cup\left(\cup_{j=1}^{m} c_{\mu}\left(p_{a j}^{-1}\left(U_{a j}\right)\right)\right) .
$$

It follows from the inverse property of the projections it follows

$$
X=p_{a}^{-1}\left(\cup_{i=1}^{n} c_{\mu}\left(U_{a i}\right) \cup \cup_{j=1}^{m}\left(c_{\mu}\left(U_{a j}\right)\right) .\right.
$$

Now

$$
X_{a}=\cup_{i=1}^{n} c_{\mu}\left(U_{a i}\right) \cup \cup_{j=1}^{m}\left(c_{\mu}\left(U_{a j}\right) .\right.
$$

From $F_{1} \cap F_{2}=\emptyset$ it follows that

$$
\cup_{i=1}^{n} c_{\mu}\left(U_{a i}\right) \cap \cup_{j=1}^{m}\left(c_{\mu}\left(U_{a j}\right)=\emptyset .\right.
$$

This is impossible since $X_{a}$ is $\mu$-connected. Thus $X=\lim \mathbf{X}$ is $\mu$-connected.

Since each open mapping has the inverse property we have the following result.

Theorem 12. Let $\mathbf{X}=\left\{X_{a}, p_{a b}, A\right\}$ be an inverse system of $w \mu$-compact spaces such that $\lim \mathbf{X}$ is $w \mu$-compact and the projections $p_{a}: \lim \mathbf{X} \rightarrow \mathbf{X}_{a}$, $a \in A$, are surjective and open. If the spaces $X_{a}$ are $\mu$-connected, then $X=\lim \mathbf{X}$ is $\mu$-connected.

\section{REFERENCES}

[1] I. Basdouria, R. Messaouda, A. Missaouia, Connected and hyperconnected generalized topological spaces, Journal of Linear and Topological Algebra, 05(04), 229-234.

[2] N. Bourbaki, Theory of sets, Herrman Publishers, 1968.

[3] Á. Császár, Generalized topology, generalized continuity, Acta Math. Hungar., 96(4) (2002), 351-357.

[4] Á. Császár, Product generalized topologies, Acta Math. Hungar., 123 (1 - 2) (2009), $127-132$.

[5] R. Engelking, General topology, PWN, Warszawa, 1977.

[6] A. S. Mashhour, A. A. Allam, F. S. Mahmoud, F. H. Khedr, On supra topological spaces, Indian J. Pure and Appl. Math., 14(4) (1983), 502-510. 
[7] W. K. Min, Some results on generalized topological spaces and generalized systems Acta Math. Hungar., 108 (2005), 171-181.

[8] M. S. Sarsak, Weakly $\mu$-compact spaces, Demonstratio Mathematica, XLV (4) (2012), 929-934.

[9] J. Thomas, S. J. John, $\mu$-Compactness in Generalized Topological Spaces, Journal of Advanced Studies in Topology, 3(3) (2012), 18-22.

[10] L. Vidyarani, M. Vigneshwaran, Some forms of $N$-closed Maps in supra Topological spaces, IOSR Journal of Mathematics(IOSR-JM), 6(4) (2013), 13-17.

[11] T. H. Yalvac, Relations between new topologies obtained from old ones, Acta Math. Hungar., 64(3) (1994), 231-235.

[12] X. Ge, Y. Ge, $\mu$-separations in generalized topological spaces, Appl. Math. J. Chinese Univ, 25 (2010), 243-252.

UNIVERSITY OF ZAGREB,

FACUlty of Organizations and Informatics,

Pavlinska 2 42000, VAraždin, Croatia

E-mail address: ivanloncar18@gmail.com 